\title{
WildPetotaDB - a database for genotype and phenotype of wild tuber-bearing species of the genus Solanum
}

\author{
Ivanova K.A. ${ }^{1 *}$, Komyshev E.G. ${ }^{1}$, Genaev M.A. ${ }^{1}$, Egorova A.A. ${ }^{1,2}$, Koloshina K.A. ${ }^{1}$, \\ Erst T.V. ${ }^{1}$, Doroshkov A.V. ${ }^{1}$, Chalaya N.A. ${ }^{3}$, Rogozina E.V. ${ }^{3}$, Ibragimova S.M. ${ }^{1}$, \\ Afonnikov D.A. ${ }^{1}$, Kochetov A.V. ${ }^{1}$, Khlestkina E.K. ${ }^{1,3}$, Gerasimova S.V. ${ }^{1}$ \\ ${ }^{1}$ Institute of Cytology and Genetics, SB RAS, Novosibirsk, Russia \\ ${ }^{2}$ Novosibirsk State University, Novosibirsk, Russia \\ ${ }^{3}$ N.I. Vavilov All-Russian Institute of Plant Genetic Resources (VIR), St. Petersburg, Russia \\ *e-mail:ivanova@bionet.nsc.ru
}

The development of high throughput phenotyping platforms is necessary to satisfy the increasing demand in fast and precise introduction of desired traits into elite crop material. In ICG, a range of high-performance phenotyping technologies is being developed. These methods allow one to characterize a large number of plants in a short period and avoid the subjective description of plants by man. Most of these methods are based on image processing and allow one to reveal stable phenotypic characteristics and quantitatively describe all phenotypic diversity. The WildPetotaDB database is being created for digital phenotypic data obtained from different samples of wild potato species from VIR collection. The purpose of creating this database is to evaluate and apply the developed digital phenotyping methods for wild potato material and provide a useful tool of optimal experimental sample selection for potato breeders and biotechnologists. The data deposited in the WildPetotaDB include the following fields for each sample: general description with photo, growing conditions, general tuberization ability, set of tuber morphological characteristics, pubescence characteristics, tuber starch content and morphology of starch granules, steroidal glycoalkaloid content and microsatellite markers of each sample. The database is being developed in a way to collect the most relevant information about the accessions, necessary for their further involvement to potato next-generation breeding programs.

Acknowledgements: This work was supported by RFBR (18-316-00068). The database was implemented on the equipment of the Bioinformatics Shared Access Center with the support of State Budget Project 0324-2019-0040. 